\title{
Holographic data storage in a DX-center material
}

\author{
I. R. Redmond and R. A. Linke \\ NEC Research Institute, 4 Independence Way, Princeton, New Jersey 08540
}

E. Chuang and D. Psaltis

Department of Electrical Engineering, Mail Stop 116-81, California Institute of Technology, Pasadena, California 91125

Received March 27, 1997

We report on the optical storage of digital data in a semiconductor sample containing DX centers. The diffraction efficiency and the bit-error-rate performance of multiplexed data images are shown to agree well with a simple model of the material. Uniform storage without an exposure schedule is demonstrated. The volume sensitivity is found to be $\sim 10^{3}$ times that of $\mathrm{LiNBO}_{3}: \mathrm{Fe}$. The importance of coherent addition of scattered light with diffracted light in holographic data storage is discussed. (C) 1997 Optical Society of America

The use of bulk photoresponsive materials for the storage of massive amounts of information by use of holographic techniques was proposed as early as 1963 by van Heerden, who showed that, in principle, a binary bit of information can be stored in a volume approximately equal to a cubic wavelength in the medium. ${ }^{1}$ Considerable interest has been shown in photorefractive materials for this application; the most widely studied of these materials is iron-doped lithium niobate $\left(\mathrm{LiNbO}_{3}: \mathrm{Fe}\right){ }^{2}$ Although this material has permitted impressive demonstrations of holographicstorage systems (e.g., Ref. 3), it has several severe limitations, including a rather small refractive-index change $\left(\Delta n \sim 10^{-4}\right)$ on exposure to light and a very poor sensitivity, typically $\sim 10^{-5} \mathrm{~cm}^{3} / \mathrm{J}$.

Semiconductor materials containing deep donor states known as DX centers were recently shown to exhibit strong optically induced changes in refractive index, which are persistent at a sufficiently low temperature $^{5-7}$ The origin of this response involves the optical release of electrons from the DX states and the resulting change of the local free-carrier density. The rate of electron recapture back into the DX state is governed by thermal activation over an energy barrier, and thus sample temperature determines the persistence time of the index change, which in practice is adjustable from years to less than a microsecond. Recently, new DX materials with higher persistence temperatures were identified, ${ }^{8}$ indicating promise for the future application of these materials to storage systems.

Here we report on a series of experiments aimed at investigating the holographic-data-storage properties of DX-center-based photoresponsive materials. The sample used in these experiments was a crystal of the compound III-V semiconductor AlGaAs doped with tellurium at a concentration of $8 \times 10^{17} \mathrm{~cm}^{-3}$. The sample was $345 \mu \mathrm{m}$ thick and had an area of approximately $5 \mathrm{~mm} \times 7 \mathrm{~mm}$. Both faces were antireflection coated, resulting in $\sim 1 \%$ reflection per surface. The sample was held in a variable-temperature cryostat and cooled in the dark to $40 \mathrm{~K}$ to sensitize it by forcing the dopant atoms into the DX states. At this tempera- ture, photoinduced persistence time is projected to be many years. The saturated change in refractive index between the dark-cooled and the fully ionized samples was measured to be $\Delta n_{\text {sat }}=0.0020$, and the exposure required for $90 \%$ of saturation is $3.0 \mathrm{~mJ} / \mathrm{cm}^{2}$, which corresponds to a volume exposure sensitivity $S$ of $1.1 \times 10^{-2} \mathrm{~cm}^{3} / \mathrm{J}$, or 1000 times that of $\mathrm{LiNbO}_{3}: \mathrm{Fe}$.

Digital information was stored in the crystal in the form of holographic recordings of "pages" of twodimensional intensity-modulated data bits. ${ }^{9}$ We used peristrophic multiplexing, ${ }^{10}$ in which the data beam is normally incident upon the hologram and the possible reference-beam directions form a cone around the object beam (Fig. 1). In these experiments we stored as many as 100 holograms, whereas fewer than 10 would have been possible with the conventional anglemultiplexing technique. ${ }^{3}$

Exposures were made with a 5-mW, 847-nm laser diode, which was expanded, filtered, and split at the rotation arm (Fig. 1). The transmitted beam is modulated by a fixed data mask and imaged onto the sample. The mask was a conventional chrome photomask containing a regular array of $96-\mu \mathrm{m}$ squares, which were randomly transparent (binary 1 ) or opaque (0), each with $50 \%$ probability. The reference beam was directed along the rotation arm and toward the sample, meeting it at $30^{\circ}$ to the normal. To record multiple pages, we rotated the arm about the sample normal and moved the data mask so that different images were recorded. On readout, the diffracted light from the sample was imaged by a $4-f$ lens system onto

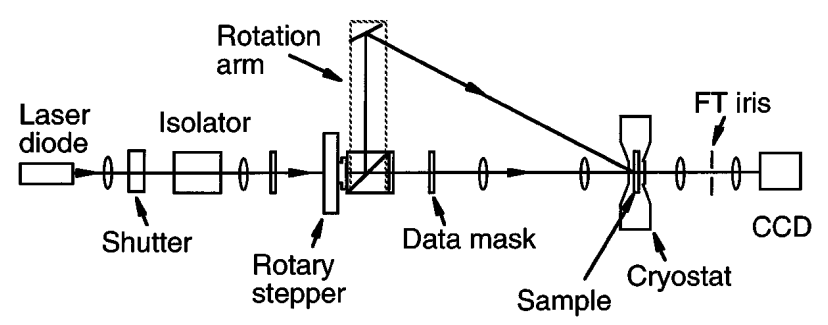

Fig. 1. Optical setup for holographic recording and reading by peristrophic multiplexing. ${ }^{3}$ FT, Fourier transform. 
a cooled CCD camera with $24-\mu \mathrm{m}$-square pixels. Any unwanted diffracted pages were blocked by an iris in the intermediate Fourier-transform plane. The holographic exposures were made and analyzed at $40 \mathrm{~K}$, and we erased and resensitized the sample by increasing its temperature to $150 \mathrm{~K}$ and dark cooling it again to $40 \mathrm{~K}$.

The relation between $\eta$ and exposure was found to match accurately that expected from an exponentially saturating response: An initial rise in $\eta$ (proportional to the square of the fluence) was followed by a drop as the DX centers became depleted and the strength of the fundamental grating fell. For this sample the peak diffraction efficiency of a single hologram was $45 \%$, which corresponds to an M/\# (Ref. 11) of 0.67 . Here we use relative diffraction efficiency; we obtained the absolute diffraction efficiency by multiplying our values by the transmission $T$ of the sample, which was in the range $0.3-0.9$ after exposure.

We performed experiments to investigate the tradeoff between peak efficiency and exposure for several series of multiplexed holograms, and the results are summarized in Table 1 for various numbers of holograms, $M$. Included in the table is the expected efficiency, $\eta_{t}$, derived from the transmission volumegrating-efficiency relation ${ }^{12}$

$$
\eta_{t}=\sin \left[\frac{\pi \Delta n t}{\lambda\left(\cos \theta_{i} \cos \theta_{o}\right)^{1 / 2}}\right]^{2}
$$

where $t$ is the hologram thickness, $\lambda$ is the wavelength in vacuum, and $\theta_{i}$ and $\theta_{o}$ are the incident and the diffracted angles, respectively. We assume that the optimum $\Delta n$ for each hologram in a multiplexed set is simply the value for an optimum single-exposure hologram divided by $M$. The dependence of efficiency on number of exposures in these measurements is consistent with the model of the holographic process in DX materials as a local phenomenon. ${ }^{13}$ In this model the index change at each point in the volume is considered to depend only on the total exposure at that point and to be independent of the exposure at neighboring points and of the time or sequence involved in reaching that final exposure. This in turn implies that a sequence of multiplexed equal exposures will yield holograms with the same diffraction efficiency. When saturation of the index change occurs, it is expected to reduce the efficiency of all the holograms equally. This was confirmed experimentally, as illustrated by Fig. 2, which shows the measured diffraction efficiencies of a series of 100 multiplexed holograms, each with an exposure of $26 \mu \mathrm{J} / \mathrm{cm}^{2}$. The uniformity of the holograms is seen to be $\pm 20 \%$, roughly half of which is attributable to the systematic variation in exposure with rotation-arm position. This uniformly saturating property of the DX materials makes following an exposure schedule when writing many holograms, as is done with photorefractives ${ }^{14}$ unnecessary.

We investigated the bit-error-rate (BER) performance of this sample by capturing images of the diffracted pages on a cooled CCD camera, finding the optimum global threshold, and analyzing the distributions of the powers in the reconstructed pixels. Since there were typically no errors observed in the $\sim 1700$ pixels within the sample area, we estimated BER's by fitting the data distribution to the Gaussian function and extrapolating. First, we found the baseline capability of the system by imaging the data mask directly through the sample onto the camera. The resulting distribution of pixel values had a signalto-noise ratio (SNR) of $\sim 200$ and a corresponding estimated BER of $10^{-45}$. The SNR is defined here as ${ }^{15}$

$$
\mathrm{SNR}=\frac{\left(\mu_{1}-\mu_{0}\right)^{2}}{\sigma_{1}^{2}+\sigma_{0}^{2}},
$$

where $\mu_{1}$ and $\mu_{0}$ are the mean 1 and 0 levels and $\sigma_{1}{ }^{2}$ and $\sigma_{0}{ }^{2}$ are the corresponding variances. The BER can be estimated for two Gaussian distributions of equal area ${ }^{15}$ :

$$
\mathrm{BER} \approx \frac{1}{2} \operatorname{erfc}\left(\frac{Q}{\sqrt{2}}\right)
$$

where

$$
Q=\frac{\mu_{1}-\mu_{0}}{\sigma_{1}+\sigma_{0}}
$$

In practice $\mu_{0}$ was not 0 but typically $2.5 \%$ of $\mu_{1}$. This result was due to the finite optical density of the mask (1.6). The finite width of the distributions (and the SNR) was found to be predominantly the result of the spatial variation of the irradiance of the beam.

Next a single page was recorded at $1 \mathrm{~mJ} / \mathrm{cm}^{2}$ and analyzed. A histogram of these data is shown in Fig. 3. The SNR here is $\sim 50$, and the estimated BER, $10^{-21}$. This is poorer than the direct maskimage result, primarily because of the holographic addition of the spatial variations on the reference beam. Thus we can consider $10^{-21}$ to be the estimated BER measurement limit of our holographic test system.

As exposures decreased in the single-hologram case, the estimated BER increased, reaching $10^{-5}$ at

Table 1. Peak Diffraction Efficiency of Multiplexed Holograms

\begin{tabular}{cccc}
\hline $\begin{array}{c}\text { Number of } \\
\text { Holograms }(M)\end{array}$ & $\begin{array}{c}\text { Exposure } \\
\left(\mu \mathrm{J} / \mathrm{cm}^{2}\right)\end{array}$ & $\eta\left(\times 10^{-6}\right)$ & $\eta_{t}\left(\times 10^{-6}\right)$ \\
\hline 20 & 180 & 950 & 990 \\
50 & 100 & 113 & 163 \\
100 & 72 & 27 & 40 \\
\hline
\end{tabular}

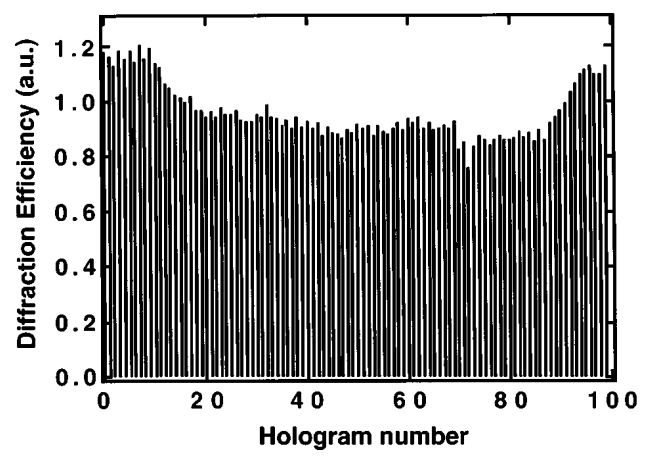

Fig. 2. Diffraction efficiency hologram number for 100hologram exposure in an AlGaAs:Te sample. All exposure values were $26 \mu \mathrm{J} / \mathrm{cm}^{2}$; i.e., no exposure schedule was used. 


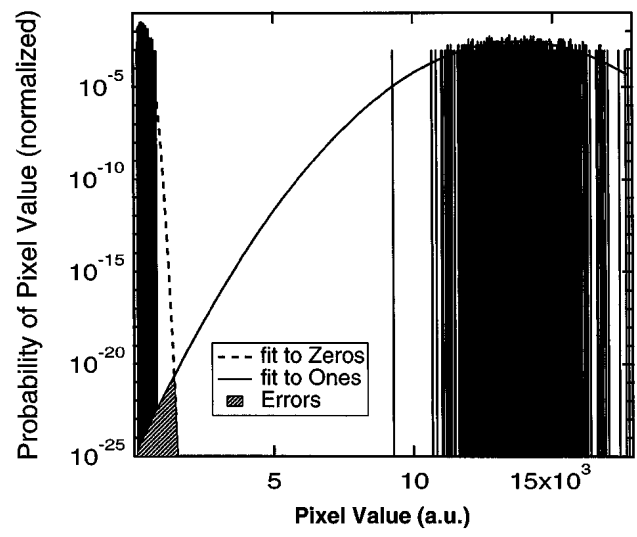

Fig. 3. Frequency of occurrence of pixel values plotted versus pixel values in arbitrary units for a strong hologram $\left(1 \mathrm{~mJ} / \mathrm{cm}^{2}\right)$. The projected BER derived from these data is $10^{-21}$.

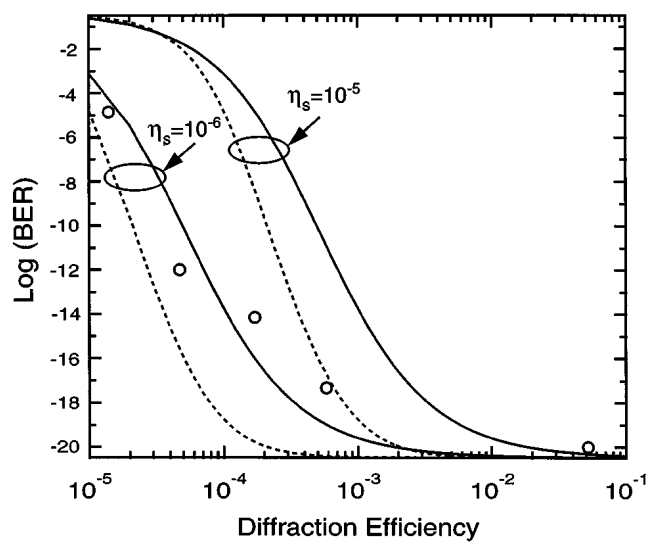

Fig. 4. Theoretical BER's for coherent (solid curves) and incoherent (dashed curves) addition of scattered light. Measurements (circles) agree reasonably well with the coherent addition model for the observed scattering level of $10^{-6}$.

$5 \mu \mathrm{J} / \mathrm{cm}^{2}$ per exposure. The increasing noise is seen as an increase in the spread of both the 0 and the 1 distributions. Even at this exposure level the diffracted light power was sufficiently far above system noise levels that we would not expect such an increase in BER. The origin of this noise was found to be coherent addition of the diffracted light and the light scattered from imperfections on the sample surface. Inclusion of the scattered light fraction $\eta_{S}$, which we assume to have random phase, leads to the following modification to $Q$ [Eq. (4)]:

$$
Q^{\prime}=\frac{\eta \sqrt{2}}{\left(\eta_{s}^{2}+2 \eta^{2} \sigma_{0}^{2}\right)^{1 / 2}+\left[\eta\left(\eta_{s}+2 \eta \sigma_{1}^{2}\right)\right]^{1 / 2}} .
$$

Using values of $\sigma_{0}=0.0086$ and $\sigma_{1}=0.098$ experimentally obtained from the strong-hologram case, in Fig. 4 we plot curves for BER versus diffraction efficiency for two scattering levels, $\eta_{S}$. Also shown in the figure are measured data points for our sample, which appear to be in good agreement with the theory for $\eta_{S}=10^{-6}$, which is also the typical value measured independently from scatter-only images.
Finally, we investigated the effect of multiple exposures on error rate by measuring the BER of a single hologram as additional holograms were superimposed at different peristrophic angles. Each exposure was $18 \mu \mathrm{J} / \mathrm{cm}^{2}$, and the diffraction efficiency of the probed page decreased from an initial value of $9.5 \times 10^{-5}$ to $1.3 \times 10^{-5}$ as the successive exposures pushed the material into saturation (with a final total exposure of $4.4 \mathrm{~mJ} / \mathrm{cm}^{2}$ ). The BER increased from $10^{-10}$ to $10^{-8}$ as the additional holograms were added. A degradation in BER similar to this is expected from the drop in $\eta$ alone. This suggests that interpage cross talk is not significant in these experiments.

In conclusion, we have demonstrated holographic storage of digital data in a semiconductor sample containing DX centers. The diffraction efficiency and the BER performance of multiplexed data images are shown to agree well with a simple model of the material in which the index change at each point in the volume depends only on the total exposure at that point and is independent of that at neighboring points and of the time or sequence involved in reaching the final exposure. This implies that a sequence of multiplexed equal exposures will yield holograms with the same diffraction efficiency, as we have confirmed experimentally. When saturation of the index change is present it appears as a reduction of the efficiency of all the holograms equally. Thus no exposure schedule is required with these materials. The recording sensitivity of our AlGaAs sample was shown to be $10^{3}$ times better than that of $\mathrm{LiNbO}_{3}: \mathrm{Fe}$. BER estimates revealed no error-rate floor intrinsic to the storage material but showed that the coherent addition of stray scattered light with the diffracted signal (a potential problem for any holographic storage system) can increase error rates well beyond those expected for incoherent background light at the same level.

\section{References}

1. P. J. Van Heerden, Appl. Opt. 2, 393 (1963).

2. D. Psaltis and F. Mok, Sci. Am. 273(5), 70 (1995).

3. F. Mok, Opt. Lett. 18, 915 (1993).

4. C. Chen, D. Kim, and D. von der Linde, Appl. Phys. Lett. 34, 321 (1979).

5. R. A. Linke, T. Thio, J. D. Chadi, and G. E. Devlin, Appl. Phys. Lett. 65, 16 (1994).

6. R. L. MacDonald, R. A. Linke, J. D. Chadi, T. Thio, G. E. Devlin, and P. Becla, Opt. Lett. 19, 2131 (1994).

7. D. D. Nolte, J. Appl. Phys. 79, 7514 (1996).

8. A. I. Ryskin, A. S. Shecheulin, B. Koziarska, J. M. Langer, A. Suchocki, I. I. Buczinskaya, P. P. Fedorov, and B. P. Sobolev, Appl. Phys. Lett. 67, 31 (1995).

9. L. Hesselink and M. Bashaw, Opt. Quantum Electron. 25, 611 (1993).

10. K. Curtis, A. Pu, and D. Psaltis, Opt. Lett. 19, 993 (1994).

11. F. H. Mok, G. W. Burr, and D. Psaltis, Opt. Lett. 21, 896 (1996).

12. H. Kogelnik, Bell Syst. Tech. J. 48, 2909 (1969).

13. R. L. MacDonald, R. A. Linke, G. E. Devlin, and M. Mizuta, Opt. Lett. 20, 1322 (1995).

14. K. Bløtekjaer, Appl. Opt. 18, 57 (1979).

15. B. E. Saleh and M. C. Teich, Fundamentals of Photonics (Wiley, New York, 1991), p. 905. 